\title{
Abelianization of First Class Constraints
}

\author{
F. Loran团 \\ Department of Physics, Isfahan University of Technology (IUT) \\ Isfahan, Iran, \\ and \\ Institute for Studies in Theoretical Physics and Mathematics (IPM) \\ P. O. Box: 19395-5531, Tehran, Iran.
}

\begin{abstract}
We show that a given set of first class constraints becomes abelian if one maps each constraint to the surface of other constraints. There is no assumption that first class constraints satisfy a closed algebra. The explicit form of the projection map is obtained at least for irreducible first class constraints. Using this map we give a method to obtain gauge fixing conditions such that the set of abelian first class constraints and gauge fixing conditions satisfy the symplectic algebra.
\end{abstract}

*e-mail: loran@cc.iut.ac.ir 


\section{Introduction}

It is known that first class constraints are responsible for the appearance of gauge freedom [1]. Given a set of first class constraints, the generator of gauge transformation is a nontrivial combination of first class constraints that satisfies some conditions derived in reference [2]. This combination is the simplest if first class constraints are abelian i.e. when the Poisson brackets of these constraints with each other vanish identically.

Abelianization of first class constraints has two more important consequences. First, following Dirac's arguments, quantization of a set of first class constraints $\phi_{i}$ 's satisfying a closed algebra,

$$
\left\{\phi_{i}, \phi_{j}\right\}=C_{i j k} \phi_{k}
$$

in which the structure functions $C_{i j k}$ 's are not $c$-numbers requires a definite operator ordering [1]. That is because in Dirac quantization, physical states are defined as null eigenstates of the operators $\hat{\phi}_{i}$ 's,

$$
\left.\hat{\phi}_{i} \mid \text { phys }\right\rangle=0,
$$

in which the operator $\hat{\phi}_{i}$ 's are defined corresponding to the constraints $\phi_{i}$ 's. Definition (2) and the algebra (11) are consistent if the operators $\hat{C}_{i j k}$ 's, defined corresponding to the structure functions $C_{i j k}$ 's, sit on the left of the operators $\hat{\phi}_{i}$ 's similar to Eq.(这). The existence of such an operator ordering is not evident generally. Apparently, when first class constraints are abelian, no such operator ordering should be considered. Second, in BRST formalism, the algebra (11), in general, leads to a complicated expansion of the BRST charge in terms of the ghosts. When first class constraints are abelian, the generator of BRST transformation can be recognized in the most simple way. For details see [3.

Abelianization procedure can be realized via constraint resolution [3, 4] or via generalized canonical transformation for general non-abelian constraints (that satisfy a closed algebra) [5]. In reference [6] the authors study abelianization via Dirac's transformation. In this method, one assumes that linear combinations of non-abelian first class constraints (satisfying a closed algebra) exist that converts the given set of non-abelian constraints to an equivalent set of abelian constraints. In this way the problem of abelianization is led to that of solving a certain system of first order linear differential equations for the coefficients of these linear combinations.

In present work, in section 2, we show that, in general, if one maps each first class constraint to the surface of the other constraints, the resulting first class constraints are abelian. The advantageous of this result is that since the explicit form of the above mentioned map can be obtained, one can practically and globally make an arbitrary set of first class constraints abelian. Of course our method is applicable only when constraints 
belong to $\mathcal{F}$, the set of "well behaved functions" of phase space coordinates. Here, by "well behaved function" we mean those functions that satisfy the validity conditions of Cauchy Kowalevski theorem [7]. Section 3 is devoted to conclusion and some discussions.

\section{The Method of Abelianization}

Cauchy Kowalevski theorem implies that for a given function $\phi \in \mathcal{F}$, the partial differential equation,

$$
\{\phi, \Omega\}=\sum_{i} a^{\mu} \frac{\partial}{\partial z^{\mu}} \Omega=1,
$$

in which $a^{\mu}=\left\{\phi, z^{\mu}\right\}$ and $z^{\mu}$ 's are the phase space coordinates, has at least one solution. To obtain $\Omega$ conjugate to a given $\phi$, i.e. $\{\phi, \Omega\}=1$, it is sufficient to find a function $H \in \mathcal{F}$, such that $\hat{\phi}^{M+1} H=0$ for an integer $M \geq 1$, where the operator $\hat{\phi}$ is defined via the relation

$$
\hat{\phi} \xi \equiv\{\phi, \xi\}, \quad \xi \in \mathcal{F} .
$$

One can show that the coefficient of $\hat{\phi}^{M} H$ in $\hat{\phi}^{M-1} H$ is a solution of Eq.(3) as follows [8]. Since $\Omega$ exists and satisfies Eq.(3), there is a local coordinate transformation $z^{\mu} \rightarrow$ $Z^{\mu^{\prime}}, \Omega, \phi$, and the function $H\left(z^{\mu}\right)=H\left(z^{\mu}\left(\Omega, \phi, Z^{\mu^{\prime}}\right)\right)$ can be written as a polynomial in $\Omega$,

$$
\begin{aligned}
& H\left(z^{\mu}\left(\Omega, \phi, Z^{\mu^{\prime}}\right)\right)=\sum_{m=0} \frac{A_{m}\left(0, \phi, Z^{\mu^{\prime}}\right)}{m !} \Omega^{m}, \\
& \hat{\phi} A_{m}=\frac{\partial}{\partial \Omega} A_{m}\left(0, \phi, Z^{\mu^{\prime}}\right)=0, \quad m \geq 0 .
\end{aligned}
$$

Comparing $\hat{\phi}^{M} H=A_{M}$ with $\hat{\phi}^{M-1} H=A_{M} \Omega+A_{M-1}$, one can determine $\Omega$ as the coefficient of $\hat{\phi}^{M} H$ in $\hat{\phi}^{M-1} H$. It should be noted that if $A_{M-1}=\tilde{A} A_{M}+A_{M-1}^{0}$ then $\Omega$ obtained in this way becomes equal to the assumed $\Omega\left(\Omega_{s}\right)$ plus $\tilde{A}$. Since $\hat{\phi} \tilde{A}=0$, the obtained $\Omega$, though is not equal to $\Omega_{s}$, but satisfies Eq.(3).

Let's define the operator $\hat{P}_{\phi}$,

$$
\hat{P}_{\phi} \equiv e^{\phi \hat{\Omega}}=\sum_{n=0} \frac{1}{n !} \phi^{n} \hat{\Omega}^{n}
$$

which satisfies the property,

$$
\hat{\Omega} \hat{P}_{\phi}=0,
$$

The validity of Eq.(8) can be directly checked using the general properties of Poisson brackets as follows.

$$
\begin{aligned}
\hat{\Omega} \hat{P}_{\phi} & =\hat{\Omega} \sum_{n=0} \frac{1}{n !} \phi^{n} \hat{\Omega}^{n} \\
& =\sum_{n=0} \frac{1}{n !}\left(n\{\Omega, \phi\} \phi^{n-1} \hat{\Omega}^{n}+\phi^{n} \hat{\Omega}^{n+1}\right) \\
& =0
\end{aligned}
$$


where in the last equality we have used $\{\Omega, \phi\}=-1$.

Lemma 1. The operator $\hat{P}_{\phi}$ is the projection map to the subspace of phase space defined by $\phi=0$, i.e. for any $\xi \in \mathcal{F}, \hat{P}_{\phi} \xi=\left.\xi\right|_{\phi}$.

Proof. From Eq.(8) and definition (7), one verifies that $\hat{P}_{\phi}^{2}=\hat{P}_{\phi}$, i.e. $\hat{P}_{\phi}$ is a projection map. Since $\phi$ and $\Omega$ are conjugate $(\{\phi, \Omega\}=1)$, there exist a (local) coordinate transformation $z^{\mu} \rightarrow Z^{\mu^{\prime}}, \phi, \Omega$, such that $\xi(z)=\tilde{\xi}(\phi, \Omega, Z)$ and $\hat{\Omega}=-\frac{\partial}{\partial \phi}=-\partial_{\phi}$. Consequently,

$$
\hat{P}_{\phi} \xi(z)=e^{-\phi \partial_{\phi}} \tilde{\xi}(\phi, \Omega, Z)=\tilde{\xi}(0, \Omega, Z)=\left.\xi\right|_{\phi}
$$

Lemma 2. Considering a function $\xi \in \mathcal{F}$ and a conjugate pair of functions $\phi$ and $\Omega$, we have

$$
\xi=\left.\xi\right|_{\phi} \quad \text { iff } \quad \hat{\Omega} \xi=0
$$

\section{Proof.}

a) If $\xi=\left.\xi\right|_{\phi}$ then from lemma $1, \xi=\hat{P}_{\phi} \xi$. Therefore using Eq.(8), $\hat{\Omega} \xi=\hat{\Omega} \hat{P}_{\phi} \xi=0$.

b) if $\hat{\Omega} \xi=0$ then $\xi=\hat{P}_{\phi} \xi=\left.\xi\right|_{\phi}$.

\section{Corollary 1.}

$$
\left\{\left.\xi\right|_{\phi},\left.\zeta\right|_{\phi}\right\}=\left\{\left.\xi\right|_{\phi},\left.\zeta\right|_{\phi}\right\}_{\phi}
$$

\section{Corollary 2.}

$$
\left\{\left.\xi\right|_{\phi}, \phi\right\}=\left\{\left.\xi\right|_{\phi}, \phi\right\}_{\phi}
$$

These corollaries can be proved using the Jaccobi identity to show that the Poisson brackets of the LHS of Eqs.(12, 13) with $\Omega$ is vanishing.

Lemma 3. If $\phi=\left.\phi\right|_{\psi}$ then $\psi=\left.\psi\right|_{\phi}$.

Proof. Since there exist a function $\Omega$ conjugate to $\phi$, using the operator $\hat{P}_{\phi}$, one can write $\psi$ as a polynomial in $\phi$ (similar to Eqs.(5),6)),

$$
\psi=\sum_{i=1} a_{i} \phi^{i}+\left.\psi\right|_{\phi}
$$

where $\hat{\Omega} a_{i}=0, i \geq 1$. If $a_{i}$ 's do not vanish, the assumption $\phi=\left.\phi\right|_{\psi}$ implies that $\psi(\phi)=0$. Thus if $\psi \neq 0$, then $a_{i}$ 's should vanish and $\psi=\left.\psi\right|_{\phi}$.

Lemma 4. If

$$
\left\{\phi_{1}, \Omega_{1}\right\}=\left\{\phi_{2}, \Omega_{2}\right\}=1
$$

and

$$
\begin{gathered}
\phi_{2}=\left.\phi_{2}\right|_{\phi_{1}}, \\
\Omega_{2}=\left.\Omega_{2}\right|_{\phi_{1}},
\end{gathered}
$$

then the operators $\hat{P}_{1}$ and $\hat{P}_{2}$ defined corresponding to the functions $\phi_{1}$ and $\phi_{2}$ (see Eq.(17)) commute with each other. 
Proof. It is sufficient to show that the operators $\phi_{1} \hat{\Omega}_{1}$ and $\phi_{2} \hat{\Omega}_{2}$ commute i.e.

$$
\left[\phi_{1} \hat{\Omega}_{1}, \phi_{2} \hat{\Omega}_{2}\right]=0 .
$$

First we show that

$$
\left[\hat{\Omega}_{1}, \phi_{2} \hat{\Omega}_{2}\right]=0 .
$$

Considering a function $\xi \in \mathcal{F}$, one verifies that,

$$
\begin{aligned}
\left(\hat{\Omega}_{1}\left(\phi_{2} \hat{\Omega}_{2}\right)\right) \xi & =\left\{\Omega_{1},\left\{\phi_{2}\left\{\Omega_{2}, \xi\right\}\right\}\right. \\
& =\phi_{2}\left\{\Omega_{1},\left\{\Omega_{2}, \xi\right\}\right\} \\
& =\phi_{2}\left\{\Omega_{2},\left\{\Omega_{1}, \xi\right\}\right\} \\
& =\left(\phi_{2} \hat{\Omega}_{2}\right) \hat{\Omega}_{1} \xi
\end{aligned}
$$

The first equality is the result of definition (14). Second equality is obtained because $\left\{\phi_{2}, \Omega_{1}\right\}=0$ as a result of Eq.(16) and lemma 2. Third equality can be derived using the Jaccobi identity and noting that from lemma 2 and Eq.(17) we have $\left\{\Omega_{1}, \Omega_{2}\right\}=0$.

From lemma 3 and Eq.(16) one verifies that

$$
\phi_{1}=\left.\phi_{1}\right|_{\phi_{2}},
$$

thus $\left\{\phi_{1}, \Omega_{2}\right\}=0$ which, using the Jaccobi identity, gives,

$$
\left[\phi_{1}, \hat{\Omega}_{2}\right]=0 .
$$

lemma 5. Given a set of first class constraints $\phi_{i}$ 's satisfying the algebra (10), there exist a function $\Omega_{i}$ that satisfies the following properties:

$$
\begin{aligned}
\left\{\phi_{i}, \Omega_{i}\right\} & =1, \\
\Omega_{i} & =\left.\Omega_{i}\right|_{\mathcal{M}},
\end{aligned}
$$

where by $\mathcal{M}$ here, we mean the surface of all constraints $\phi_{i}$ 's.

Proof. The function $\Omega_{i}$ with the above properties can be determined if one finds a function $H=\left.H\right|_{\mathcal{M}}$ such that $\hat{\phi}_{i} H \neq 0$ but $\hat{\phi}_{i}^{M+1} H=0$ for some integer $M$. See the arguments below Eq.(医) and note that since $H=\left.H\right|_{\mathcal{M}}$ the function $\Omega_{i}$ obtained in this way satisfies Eq.(24). Of course one should show that there exist a function $H=\left.H\right|_{\mathcal{M}}$ such that $\left\{\phi_{i}, H\right\} \neq 0$. To prove this assertion, assume that there does not exist any function with this property. Since there exist a function $\Omega_{i}$ that satisfies Eq.(23), this assumption leads to the following equality,

$$
\left\{\phi_{i},\left(\Omega_{i}-\left.\Omega_{i}\right|_{\mathcal{M}}\right)\right\}=1,
$$


because

$$
\left\{\phi_{i},\left.\Omega_{i}\right|_{\mathcal{M}}\right\}=0
$$

In addition,

$$
\Omega_{i}-\left.\Omega_{i}\right|_{\mathcal{M}}=\sum_{i} a_{i} \phi_{i}+O\left(\phi^{2}\right)
$$

in which the first term on the RHS is linear in $\phi_{i}$ 's i.e. $a_{i}=\left.a_{i}\right|_{\phi_{j}}$ and the second term includes all nonlinear terms in $\phi_{i}$ 's. Consequently Eq.(25) implies that

$$
\left\{\phi_{i},\left(\sum_{j} a_{j} \phi_{j}\right)\right\}=1 .
$$

This is not consistent with the algebra (1) because the coefficients $a_{i}$ 's do not vanish on the surface of constraints and consequently the algebra (1) can not be closed.

The above results provide a simple method for abelianization of first class constraints $\phi_{i}^{0}, i=1, \cdots, n_{0}$ satisfying the closed algebra

$$
\left\{\phi_{i}^{0}, \phi_{j}^{0}\right\}=\sum_{k} C_{i j}^{k} \phi_{k}^{0} .
$$

To obtain this result, consider one of the constraints, e.g. $\phi_{1}^{0}$ and its conjugate $\Omega_{1}^{0}$. The operator $\hat{P}_{0}=e^{\phi_{1}^{0} \hat{\Omega}_{1}^{0}}$ maps all the remaining constraints to the constraint surface $\phi_{1}^{0}=0$. Let's call these mapped constraints $\phi_{i}^{1}, i=1, \cdots, n_{1} \leq n_{0}-1$. Since $\phi_{i}^{1}=\left.\phi_{i}^{1}\right|_{\phi_{1}^{0}}$, using lemma 3 , one realizes that $\phi_{1}^{0}=\left.\phi_{1}^{0}\right|_{\mathcal{M}^{1}}$, where $\mathcal{M}^{1}$ is the constraint surface determined by the constraints $\phi_{i}^{1}$ 's. Considering $\phi_{1}^{1}$ and its conjugate $\Omega_{1}^{1}$, the operator $\hat{P}_{1}=e^{\phi_{1}^{1} \hat{\Omega}_{1}^{1}}$ maps the remaining constraints to the constraint surface $\phi_{1}^{1}=0$. One should note that the function $\Omega_{1}^{1}$ should be chosen such that $\Omega_{1}^{1}=\left.\Omega_{1}^{1}\right|_{\phi_{1}^{0}}$, because the map $\hat{P}_{1}$ should leave the constraints on the surface of $\phi_{1}^{0}$. Lemma 5 proofs the existence of such a function $\Omega_{1}^{1}$. Continuing this process, one finally ends up with a set of irreducible constraints $\phi_{1}^{0}, \phi_{1}^{1}, \cdots, \phi_{1}^{m}, m \leq n_{0}$, equivalent to the constraints $\phi_{i}^{0}$ 's, $i=1, \cdots, n_{0}$. By equivalence, we mean that the constraint surface $\mathcal{M}$ defined by the constraints $\phi_{i}^{0}$ 's is equivalent to that determined by the constraints $\phi_{1}^{a}$ 's, $a=0, \cdots, m$. We say the set of $\phi_{i}^{0}$ are equivalent to $\phi_{1}^{a}$ 's because $\phi_{1}^{a}$ 's are, by construction, some (nonlinear) combinations of $\phi_{i}^{0}$ 's.

Lemma 6 . The set of constraints $\phi_{1}^{a}$ 's, satisfy the following relation,

$$
\phi_{1}^{a}=\left.\phi_{1}^{a}\right|_{\phi_{1}^{b}}, \quad b \neq a .
$$

Proof. First we show that

$$
\phi_{1}^{a}=\left.\phi_{1}^{a}\right|_{\phi_{1}^{b}}, \quad b<a, a=1, \cdots, m .
$$

For this it is sufficient to prove that

$$
\left\{\Omega_{1}^{b}, \phi_{1}^{a}\right\}=0, \quad b<a,
$$


then the validity of Eq.(31) can be verified using lemma 2. To prove Eq.(32) we note that:

1) by construction,

$$
\phi_{1}^{a}=\hat{P}_{a-1} \cdots \hat{P}_{0} \phi_{1}^{a}
$$

in which

$$
\hat{P}_{b}=e^{\phi_{1}^{b} \hat{\Omega}_{1}^{b}}
$$

where

$$
\begin{aligned}
& \phi_{1}^{b}=\left.\phi_{1}^{b}\right|_{\phi_{1}^{c}}, \quad c<b, \\
& \Omega_{1}^{b}=\left.\Omega_{1}^{b}\right|_{\phi_{1}^{c} .} \\
& \left\{\phi_{1}^{b}, \Omega_{1}^{b}\right\}=1 .
\end{aligned}
$$

Lemma 5 guarantees the existence of $\Omega_{1}^{b}$ satisfying Eqs.(36, 37).

2) From lemma 4 and Eqs.(35) 37) we find that the operators $\hat{P}_{b<a}$ appearing in Eq. (33) commute. Eq.(8) implies the validity of Eq.(32). Using lemma 3 and Eq.(31) one verifies that

$$
\phi_{1}^{a}=\left.\phi_{1}^{a}\right|_{\phi_{1}^{b}}, \quad b>a, a=0, \cdots, m-1,
$$

which completes the proof.

Lemma 7. The set of constraints $\phi_{1}^{0}, \cdots, \phi_{1}^{m}$ are abelian.

Proof. Assume the first class constraints $\phi_{1}^{a}$, s satisfy a closed algebra

$$
\left\{\phi_{1}^{a}, \phi_{1}^{b}\right\}=\sum_{c} C_{c}^{a b} \phi_{1}^{c}
$$

for some functions $C_{c}^{a b} \in \mathcal{F}$. From Eq.([12), and the fact that $\phi_{1}^{a}=\left.\phi_{1}^{a}\right|_{\mathcal{M}^{a b}}$ and $\phi_{1}^{b}=\left.\phi_{1}^{b}\right|_{\mathcal{M}^{a b}}$ in which $\mathcal{M}^{a b}$ is the constraint surface determined by all constraints except $\phi_{1}^{a}$ and $\phi_{1}^{b}$ (see lemma 6), we have

$$
C_{c}^{a b}=0 \quad c \neq a, b
$$

In addition $\phi_{1}^{a}=\left.\phi_{1}^{a}\right|_{\phi_{1}^{b}}$ implies that $C_{b}^{a b}=0$. Similar argument shows that $C_{a}^{a b}=0$. Therefore

$$
\left\{\phi_{1}^{a}, \phi_{1}^{b}\right\}=0
$$

This result is interesting because the map between the set of (reducible) non-abelian constraints $\phi_{i}^{0}$ 's to the irreducible abelian constraints $\phi_{1}^{a}$ 's is globally defined and can be used practically. One should only choose a proper constraint at each level, where the projection operator is defined and applied. By proper we mean that, for example at first level, $\phi_{1}^{0}$ should not be a multiple of some other constraints. These difficulties disappear if first class constraints are generated by the chain by chain method [9] and consequently they are irreducible. 
Lemma 8. If $\phi=\left.\phi\right|_{\psi},\{\phi, \psi\}=0$ and $\{\phi, \Omega\}=1$, then $\left\{\phi,\left.\Omega\right|_{\psi}\right\}=1$.

Proof. Writing $\Omega$ as a polynomial in $\psi$,

$$
\Omega=\sum_{i=1} a_{i} \psi^{i}+\left.\Omega\right|_{\psi}
$$

one verifies that,

$$
1=\{\phi, \Omega\}=\sum_{i=1}\left\{\phi, a_{i}\right\} \psi^{i}+\left\{\phi,\left.\Omega\right|_{\psi}\right\}
$$

Thus,

$$
1=\left\{\phi,\left.\Omega\right|_{\psi}\right\}_{\psi}=\left\{\phi,\left.\Omega\right|_{\psi}\right\}_{\psi}=\left\{\phi,\left.\Omega\right|_{\psi}\right\},
$$

where in the third equality we have used Eq.(12).

\section{Corollary 3.}

$$
\Omega=\left.\Omega\right|_{\phi}
$$

Corollary 4. The functions $\Omega_{1}^{a}$ 's conjugate to $\phi_{1}^{a}$ 's, $\left\{\phi_{1}^{a}, \Omega_{1}^{a}\right\}=1$, satisfy the following properties,

$$
\begin{gathered}
\left\{\phi_{1}^{a},\left.\Omega_{1}^{b}\right|_{\mathcal{M}}\right\}=\delta^{a b}, \\
\left\{\left.\Omega^{a}\right|_{\mathcal{M}},\left.\Omega^{b}\right|_{\mathcal{M}}\right\}=0 .
\end{gathered}
$$

To prove this equalities use corollary 3, Eq.(11) and note that $\phi_{1}^{a}=\left.\phi_{1}^{a}\right|_{\phi_{1}^{b \neq a}}$. The proof is similar to the proof of lemma 7. This result is interesting since from Eq.(46), one verifies that $\left.\Omega_{1}^{a}\right|_{\mathcal{M}}$ can be considered as gauge fixing conditions, because the equation $\left.\Omega_{1}^{a}\right|_{\mathcal{M}}=0$ eliminates the gauge freedom generated by the first class constraint $\phi_{1}^{a}$ 's. In this way we have found first class constraints and gauge fixing conditions that satisfy symplectic algebra.

\section{Summary}

We showed that irreducible first class constraints obtained by mapping each element of a given set of first class constraints to the surface of the other elements, are abelian. The explicit form of these maps at least when constraints are irreducible are obtained. Final result only depends on general properties of Poisson bracket and do not depend on its explicit form. Consequently, if system possesses both first class and second class constraints and first class constraints do not obey a closed algebra, one can replace ordinary Poisson brackets with Dirac brackets corresponding to the second class constraints and apply the method studied here to make first class constraints abelian with respect to Dirac bracket. Furthermore, using Eq.(12) (corollary 1) one can show that if one maps the first class constraints to the surface of second class constraints, then they satisfy a closed algebra and abelianization with respect to ordinary Poisson bracket becomes straightforward. The explicit form of projection map to the surface of second class constraints is studied in [8]. 


\section{References}

[1] P. A. M. Dirac, Can. J. Math. 2, (1950) 129 ; Proc. R. Soc. London Ser. A 246, (1958) 326; "Lectures on Quantum Mechanics" New York: Yeshiva University Press, 1964 ,

[2] C. Batlle, J. Gomis, X. Gracia and J. M. Pons, J. Math. Phys. 30 (6), (1989) 1345; J. M. Pons and J. A. Garcia, Int. J. Mod. Phys. A 15 (2000) 4681, hep-th/9908151.

[3] M. Henneaux, Phys. Rep. 126, (1985) 1; M. Henneaux and C. Teitelboim "Quantization of Gauge System" Princeton University Press, Princeton, New Jersey, 1992.

[4] J. Goldberg, E. T. Newman and C. Rovelli, J. Math. Phys. 32, (1991) 2739.

[5] P. G. Bergman and I. Goldberg, Phys. Rev. 98, (1955) 531; P. G. Bergman ibid. 98, (1955) 544.

[6] S. A. Gogilidze, A. M. Khvedelidze and V. N. Pervushin, J. Math. Phys. 37, (1996) 1760, hep-th/9504153.

[7] F. John, "Partial Differential Equations", vol. 1, Fourth Edition, Springer-Verlag, New York Inc., 1981.

[8] F. Loran, "Symplectic Algebra Among Second Class Constraints", submitted to Int. J. Mod. Phys. A.

[9] F. Loran and A. Shirzad, Int.J.Mod.Phys. A17, (2002), 625, hep-th/0003010;

A. Shirzad and F. Loran, to appear in Int. J. Mod. Phys. A, hep-th/9912289. 